\title{
Open Repair of Pararenal Aneurysms: Renal Vessel Surgical Management
}

\author{
Daniele Mascia, Alessandro Grandi, \\ Luca Porcellato, Luca Bertoglio, Andrea Kahlberg, \\ Domenico Baccellieri, Germano Melissano, \\ and Roberto Chiesa
}

\subsection{Proximal Abdominal Aortic Aneurysms (P-AAA)}

The proximal abdominal aorta constitutes a particular location for the dilative process of the aneurysms because the splanchnic vessels or the renal artery ostium may be involved. As a result, we can make a more specific classification of this type of aneurysms:

- Juxtarenal infrarenal abdominal aortic aneurysms (JR-AAA): The proximal extension of the aneurysms starts adjacent or at the lower margin of the renal artery origins, without involving them in the dilative process.

- Pararenal infrarenal abdominal aortic aneurysms (PR-AAA): One or both renal arteries are involved in the dilative process.

- Suprarenal abdominal aortic aneurysms (SR-AAA): The splanchnic arteries and the renal artery origins are involved in the dilative process.

D. Mascia $(\bowtie) \cdot$ A. Grandi $\cdot$ L. Porcellato

L. Bertoglio · A. Kahlberg · G. Melissano

Vascular Surgery Unit, IRCCS San Raffaele Scientific

Institute, Milan, Italy

e-mail: mascia.daniele@hsr.it

D. Baccellieri

Unit of Vascular Surgery, San Raffaele Scientific

Institute, Milan, Italy

R. Chiesa

Unit of Vascular Surgery, San Raffaele Scientific

Institute, Vita-Salute University, Milan, Italy

\subsection{Epidemiology}

\subsubsection{Incidence}

The involvement of the proximal abdominal aorta takes place in $5-15 \%$ of all AAAs $[1,2]$. AAAs mostly affect the population above 50 years of age; they are two to six times more frequent in men than women and two to three times more common in people of Caucasian descent than in other populations [3-5]. Screening studies have provided us with important information on the incidence and prevalence of this disease: for men above 50 years of age, an incidence of 3.5 for 1000 people/year [6] was shown, while new-onset AAAs were discovered in only $2 \%$ of patients at a second examination performed after a mean time of 5.5 years, after a negative result upon first examination. Overall, the incidence by age of ruptured and symptomatic P-AAAs, and the mortality rate of P-AAAs, is six times greater in men than in women. A significant increase in the incidence of asymptomatic AAAs has been observed over the last two decades [7, 8], which may be due to increased diagnostic detection. However, there is emerging proof of a plateau reached by hospitalization numbers for aortic aneurysm repair, perhaps owing to health policies which are finalized to reduce the morbidity and mortality of cardiovascular diseases. 


\subsubsection{Prevalence}

The asymptomatic prevalence estimate of TAAA (probability of being an AAA carrier) is more accurate compared to the incidence estimate (probability of developing AAA), now that duplex ultrasound screenings are performed on numerous populations. The duplex ultrasound screening studies on the population offer the most accurate information for the prevalence of AAA $(>3 \mathrm{~cm})$. Those studies have been conducted both as epidemiologic screening studies (Tromso and Rotterdam) and as randomized studies to evaluate the benefits of the screening itself [Multicentric Aneurysm Screening Study (MASS), Chichester, Viborg, Western Australia]. In Veteran Affairs, a screening study of more than 73,000 patients between 50 and 79 years of age was carried out. The AAA $\geq 3 \mathrm{~cm}$ prevalence was $4.6 \%$, and the $\mathrm{AAA} \geq 4 \mathrm{~cm}$ prevalence was $1.4 \%$ [9]. The reported prevalence varies according to age and gender. The highest AAA $\geq 3 \mathrm{~cm}$ prevalence was $5.9 \%$ that was found in a subpopulation of white males with smoking habits between 50 and 79 years of age [10].

\subsection{Preoperative Analysis}

\subsubsection{Clinical Presentation and Indication for Surgery}

AAA is generally a pathology found by chance, as the majority of cases are found to be asymptomatic. Any symptoms related to a locoregional compression by the aneurysm are rare, as there are no structures and organs that suffer the aneurysmal dilation. Ischemic symptoms related to embolization for the crushing of the thrombus near the walls of the aneurysm are even rarer. A typical clinical sign is the appreciation of a pulsating mass in the epimesogastrium synchronous with the cardiac cycle, particularly in aneurysms of a certain size and in people of slim build. From a semiotic perspective, the suspicion of a pararenal aneurysm must be present when, during the physical examination, the upper pole of the aneurysmal dilation is not palpable at the arch rib (DeBakey maneuver). In the case of AAAs, the indication for elective surgery arises for aneurysms with a diameter $>55 \mathrm{~mm}$, even if intervention must be taken into account for smaller diameters in case of rapid growth and morphological aspects indicating a high risk of ruptures, such as highly eccentric thrombotic material, protrusions (blisters), or even parietal fissures. Currently, the diameter of the aorta is the best criteria for predicting the risk of aortic rupture [11]. According to a study by Juvonen et al., each increase of $1 \mathrm{~cm}$ in the diameter of abdominal aortic aneurysms is related to a 1.9-fold increase of the relative risk of rupture [12]. Lo et al. showed that the risk of aortic rupture is precisely calculated, by taking the area of the patient's body surface area (BSA) [13] into account.

\subsubsection{Diagnostic Imaging}

The use of imaging techniques is crucial in the assessment of AAAs, both to confirm the clinical diagnostic suspicion and to precisely define the critical morphological characteristics indicating surgery.

\subsubsection{Duplex Ultrasound}

The transabdominal ultrasonography is the least invasive examination and more frequently applied, in particular for screening and follow-up. Vessel diameter is measured by ultrasonography, allowing for an inter-operator variability of around $5 \mathrm{~mm}$ in $84 \%$ of studies, and is more accurate in assessing the anteroposterior than the transverse [14] diameter. The display of the suprarenal aorta and the iliac arteries may be impeded by anatomical characteristics, particularly in obese patients. In such cases, the ultrasound cannot accurately determine the presence of rupture [15] and is often not able to precisely determine the proximal extension of AAA [16]. Ultrasound typically underestimates the anteroposterior diameter of an AAA by 2-4 mm [17-20]. In general, ultrasound is used to diagnose and monitor the AAA until the aneurysm nears a typically operable diameter size. At this point, secondary examinations are required. 


\subsubsection{CT Angiography}

The abdominal aorta CT angiography must be performed with an acquisition thickness of $1.25 \mathrm{~mm}$ or less, depending on the available CT technology (Fig. 3.1). The overlap (in particular sections, the interval is half of the actual thickness) is recommended to increase the quality of multiplanar images (MPR) and 3D visualization. This phase, without contrast medium, allows for the evaluation of potential wall calcification or the presence of prosthetic aortic grafts in already operated patients. Aneurysms of $3-4.5 \mathrm{~cm}$ in diameter require 12-month follow-up with CT scan. This intervention is highly recommended when the diameter increases by $5 \mathrm{~mm}$ or more within 6 months or in cases of diameters larger than $55 \mathrm{~mm}$. The CT angiography has become essential for surgical planning [21]. The executive protocol of a CT with contrast enhancement at our center involves the intravenous injection of a high concentration of iodized agent (370$400 \mathrm{mg} / \mathrm{mL})$ at a high flow rate $(4-5 \mathrm{~mL} / \mathrm{s})$ to obtain a prolonged opacification of the vessels [22]. The interpretation of the CT findings is based on the analysis of a combination of axial images and reformatted images. For some years now, several software applications are available

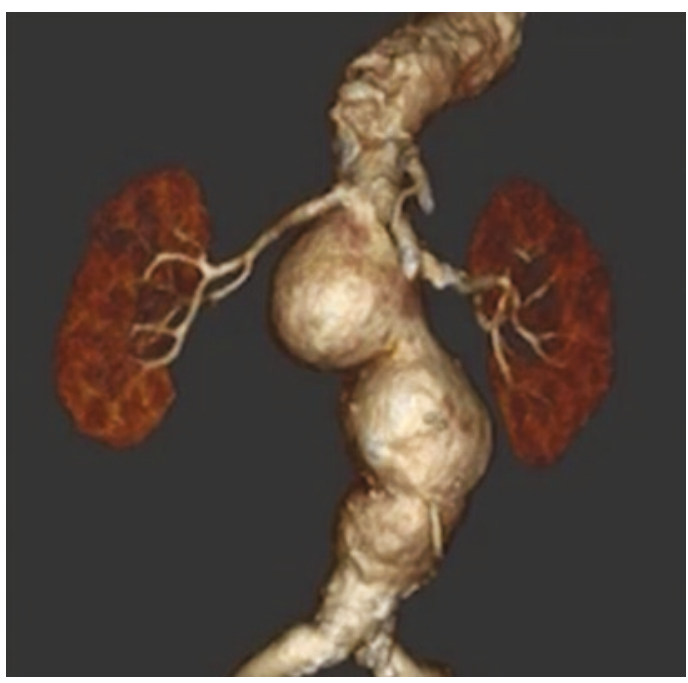

Fig. 3.1 $3 \mathrm{D}$ reconstruction of a juxtarenal infrarenal abdominal aortic aneurysm (freeware, OsiriX MD, Pixmeo; paid, TeraRecon, TeraRecon, Inc.) to view and analyze DICOM (Digital Imaging and Communications in Medicine) files, which is the most common format used in medical imaging. This software allows you to automatically download files from any DICOM CD-ROM, regardless of the hospital or the country of origin and rescale. If the acquired images are of sufficient quality, these studies allow a proper assessment, avoiding replication of the CT. The vascular surgeon, who will perform the endovascular or surgical procedure, is personally involved in the assessment of the aortic images and in planning/ sizing the case. For the pre-procedural assessment, in addition to morphological information, such as diameter and extension of the aneurysm, angiography provides other fundamental anatomical information such as:

- The quality of the aortic wall

- The involvement of visceral vessels and iliac arteries

- Clamping locations

- Left renal vein (anterior, posterior, or double)

- Visceral vessel-associated diseases (stenosis, occlusion, anatomical abnormalities, such as the "double cluster," hypoplasia)

- Extravascular-associated diseases (pelvic kidney and horseshoe kidney)

The images acquired from CT angiography are equally essential for a thorough follow-up, to recognize quickly and subsequently treat potential complications.

\subsubsection{MRI}

The absence of exposure to ionizing radiation makes the use of magnetic resonance imaging (MRI) for diagnosis and subsequent controls of P-AAAs an apparently advantageous choice compared to CT. Magnetic resonance angiography is comparable to the CT angiography in many aspects but has some shortcomings: the lack of visualization of calcified plaques-it typically owns half of the spatial resolution; it has longer acquisition times and creates more problems for claustrophobic patients, but it can be useful in 
cases of allergy to iodinated contrast. However, its high cost and limited diffusion do not make it the first choice.

\subsubsection{Preoperative Risk Stratification and Patient Optimization}

Surgical repair of a proximal abdominal aortic aneurysm is considered a high-risk intervention. Clamping the aorta carries significant hemodynamic stress, which is the reason careful assessment of cardiac, lung, and kidney function is required to determine the eligibility of patients for intervention. Often patients with abdominal aortic aneurysm have comorbidities, such as ischemic heart disease (16-30\%), respiratory problems, and kidney failure [23].

\subsubsection{Heart Function}

The European Society of Cardiology (ESC) has recently developed guidelines aimed at helping the choice for a more efficient approach to preoperative evaluation [24]. Vascular interventions are of particular interest because they will involve a significant risk of cardiac complications. These guidelines propose an algorithm to identify patients with a significant risk of cardiac complications during elective surgery. The gradual approach advocated by the ESC has more than one step. The first one is the identification of active heart disease: unstable angina pectoris, recent myocardial infarction (within 30 days), residual myocardial ischemia, acute heart failure, symptomatic valvular disease, and significant cardiac arrhythmias. The second step is the evaluation of functional capacity, which is measured in metabolic equivalents (MET). The third step is the evaluation of the specific risk for surgical procedures. Aortic surgery is considered at high risk (rate of cardiac events at 30 days $>5$ ) [25]. It is also important to assess the risk factors in patients with low functional capacity. If there are up to two risk factors, patients can proceed to the intervention, providing a treatment with statins and betablockers (including ACE inhibitors in the presence of left ventricular systolic dysfunction are needed). The fourth step is the execution of a noninvasive stress test in patients with a low functional capacity and more than two risk factors, which are scheduled for surgery.

The transthoracic echocardiography is an important noninvasive screening test that assesses the valve function. Coronary CT angiography is becoming the alternative less-invasive method for the visualization of the anatomy of the coronary arteries. The new multi-detector of CT angiography, built to reduce radiation, uses the acquisition of related ECG images allowing us to obtain images of specific phases of the cardiac cycle. In patients with asymptomatic disease, a severe occlusive disease of the coronary arteries can be treated with percutaneous transluminal angioplasty before proceeding to repair the aneurysm.

\subsubsection{Renal Function}

The renal function is traditionally calculated by measuring levels of creatinine, serum electrolytes, and blood urea nitrogen (BUN). These indices are quite sensitive, especially in patients with intermediate and moderate degrees of renal dysfunction. The National Kidney Foundation recommends the use of estimated glomerular filtration rate (GFR) to evaluate kidney function and avoid misclassification based on only one level of serum creatinine [26]. Based on the assessment of GFR, chronic kidney disease has proven to be a strong predictor factor of death after the surgical treatment of abdominal aortic aneurysms, even in patients without clinical evidence of preoperative renal disease. CT angiography, MRI, and ultrasound determine the kidneys' size and renal anatomy. The classification KDOQI has identified five levels of kidney function, based on GFR values. There is also evidence that low levels of GFR and high levels of albuminuria independently correlated with mortality, cardiovascular events, and the probability of progression to kidney failure. This is the reason the latest guidelines suggest the integration of the GFR and albuminuria study for a more accurate assessment of renal function. Of great interest are the data in a meta-analysis 
conducted by the Chronic Kidney Disease Prognosis Consortium, in which the independent and combined association of the GFR and albuminuria on cardiovascular mortality and on all-cause of death in the general population were evaluated. Compared with a GFR of $95 \mathrm{~mL} / \mathrm{min}$, the relative risk of death from all causes was increased by $18 \%$ for GFR of $60 \mathrm{~mL} / \mathrm{min}$ and $57 \%$ for GFR of $45 \mathrm{~mL} / \mathrm{min}$ and was more than tripled for GFR of $15 \mathrm{~mL} / \mathrm{min}$. Similarly, the risk-related albuminuria followed linear growth, no threshold effect, and it was already significant at low values of albuminuria or in the presence of traces of proteinuria. Similar results were also found for mortality from all causes. In conclusion, GFR and albuminuria are independent predictors and risk multipliers of mortality in the general population, with no evidence of interactions [27].

\subsubsection{Respiratory Function}

Potential risk factors for respiratory failure are age, gender, aneurysm size, preoperative lung function calculated through the forced expiratory volume in $1 \mathrm{~s}$ (FEV1), forced vital capacity (FVC), the relationship between these two units (FEV1/FVC), comorbidities and operative parameters (such as when an extended incision is requested, when there is a left hemi-diaphragm paralysis, or when there is the necessity for a high number of transfusions), timing of aortic clamping operators, and postoperative complications (in addition to the lung, even kidney, failure, bleeding, and infection of surgical wounds). Pre-, intra-, and postoperative blood tests (creatinine, BUN, lactic dehydrogenase, $\mathrm{pH})$ are also important. A standard chest X-ray reveals unexpected abnormalities in approximately $5 \%$ of patients between 40 and 60 years and in 6-30\% of patients over 60 years. The abnormalities include tracheal deviation, deviation of the left main bronchus, lung or mediastinal masses, lung bubbles, pulmonary edema, pneumonia, atelectasis, fractures of the vertebrae or ribs, cardiomegaly, and dextrocardia. The evaluation of lung function with spirometry and arterial blood gas test is used in all patients who undergo open surgery. The reduction in FEV1 or other spirometric indicators of pulmonary function not within the limits, in addition to arterial blood gas analysis abnormalities, such as hypoxemia or hypercapnia, suggests that the patient is at high risk for the development of postoperative pulmonary complications.

\subsection{Surgical Technique}

Open surgery for P-AAA is a major vascular procedure with significant mortality and morbidity rates, mostly with regard to renal function. A radial arterial pressure line is always inserted in order to guarantee continuous intraoperative arterial blood pressure monitoring. A central venous catheter (jugular or subclavian) is inserted to rapidly administer medications and fluids and to monitor central venous pressure.

Temperature is monitored by means of a bladder or nasopharyngeal sensor and is used to guide hypothermia when the hypothermic perfusion of renal arteries is required or where long-lasting procedural time or large bleeding may be expected.

Intraoperative transesophageal echocardiography (TEE) enables the dynamic monitoring of hemodynamic status assessing the systolic and diastolic function, ejection fraction, wall motion, dyssynchrony, and valvular function in patients with increased cardiac risk or who develop unexpected nonreversible intraoperative hypotension.

\subsubsection{Transperitoneal Access}

The patient is usually in a supine position. A median laparotomy from the xiphoid to the symphysis pubis is routinely performed; in patients with specific concerns such as previous median laparotomies, severe obesity, or other technical issues, bilateral subcostal laparotomy may be the alternative (Fig. 3.2). With this access, however, the distal control of iliac arteries is more challenging, and it should be avoided in cases of associated iliac aneurysms. In cases of very large aneurysms, transperitoneal medial 

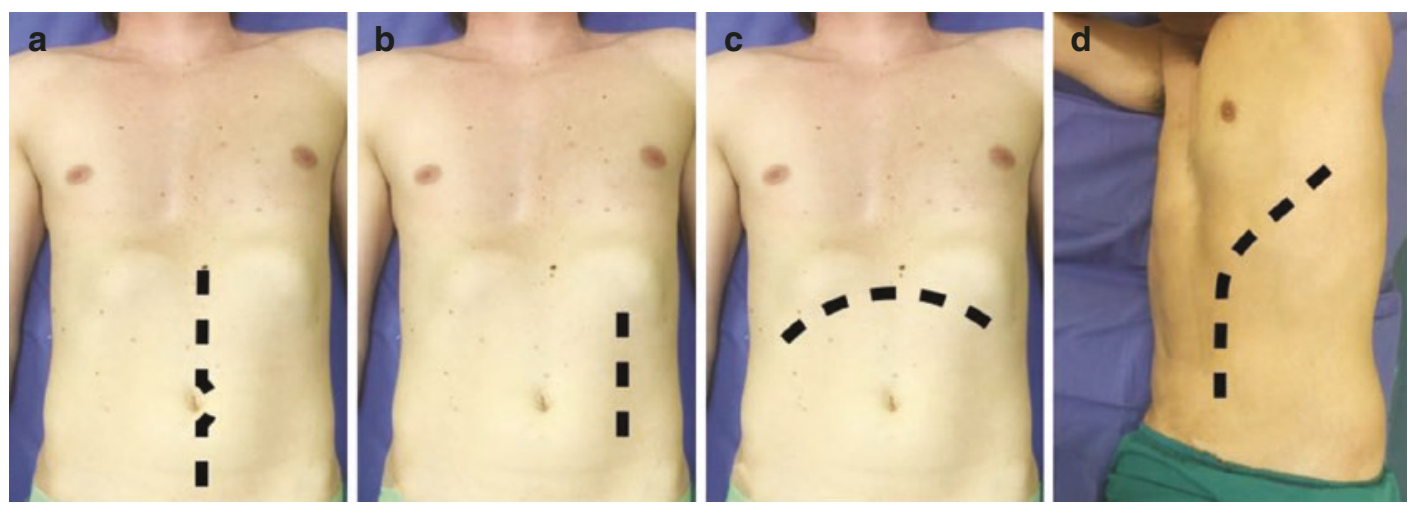

Fig. 3.2 (a) Xipho-pubic laparotomic access, (b) pararectal laparotomy, (c) bilateral subcostal laparotomy access, and (d) thoraco-phreno-laparotomy access

visceral rotation is helpful to obtain adequate exposure and proximal aortic control.

\subsubsection{Retroperitoneal Access}

The patient is positioned in partial right lateral decubitus. The left shoulder is fixed vertically, the pelvis is rotated toward the horizontal plane, and the table broken. The incision usually extends from the lateral border of the left rectus muscle anteriorly to the erector spinae in the eighth intercostal space to the area of the umbilicus. The level and the extent of the incision depend on the patient's anatomy and required exposure. In extensive pathology, a thoraco-phrenolaparotomy may be performed.

\subsubsection{Aortic Clamping and Reconstruction}

In the preparation of the pararenal aorta, correct management of the left renal vein is mandatory. Usually collocated anteriorly to the aorta, the left renal vein can be mobilized to obtain a greater proximal exposition of the aorta, dividing the gonadic, surrenali, and lumbar ramifications (Fig. 3.3). Alternatively, the left renal vein can be sectioned close to the inferior vena cava; unfortunately, this type of approach increases the postoperative renal insufficiency (Fig. 3.4).
The proximal aortic cross-clamping site is, along with aortic reconstruction technique, the most important issue in the surgical treatment of P-AAA. Suprarenal and supra-celiac clamping (Fig. 3.5) are possibilities. The correct choice depends on the proximal extension of the aneurysm, its morphology, and on the ease of exposing the suprarenal aorta. Sometimes, supraceliac clamping, especially in inflammatory aneurysm, re-treatment, and emergency treatment can be technically manageable compared to suprarenal one. The need for supra-celiac clamping must be brought forward as much as possible, based on the preoperative imaging, and the presence of extensive calcifications or thrombotic material in pararenal aorta highlighted.

Systemic heparinization is administered before aortic cross-clamping in order to achieve activated clotting time (ACT) of $>200 \mathrm{~s}$. Thromboembolism from pararenal aorta to renal arteries is a well-known complication and can be prevented by renal artery crossclamping before aortic manipulation and clamping. Common iliac arteries and aorta are then cross-clamped. The aneurysm is entered, thrombus is removed, and back-bleeding from lumbar arteries is quickly controlled by the ligation of the ostia (2/0 polypropylene suture reinforced by Teflon pledgets). If the inferior mesenteric artery is patent, it is temporarily clamped with a bulldog clamp, as previously described. 


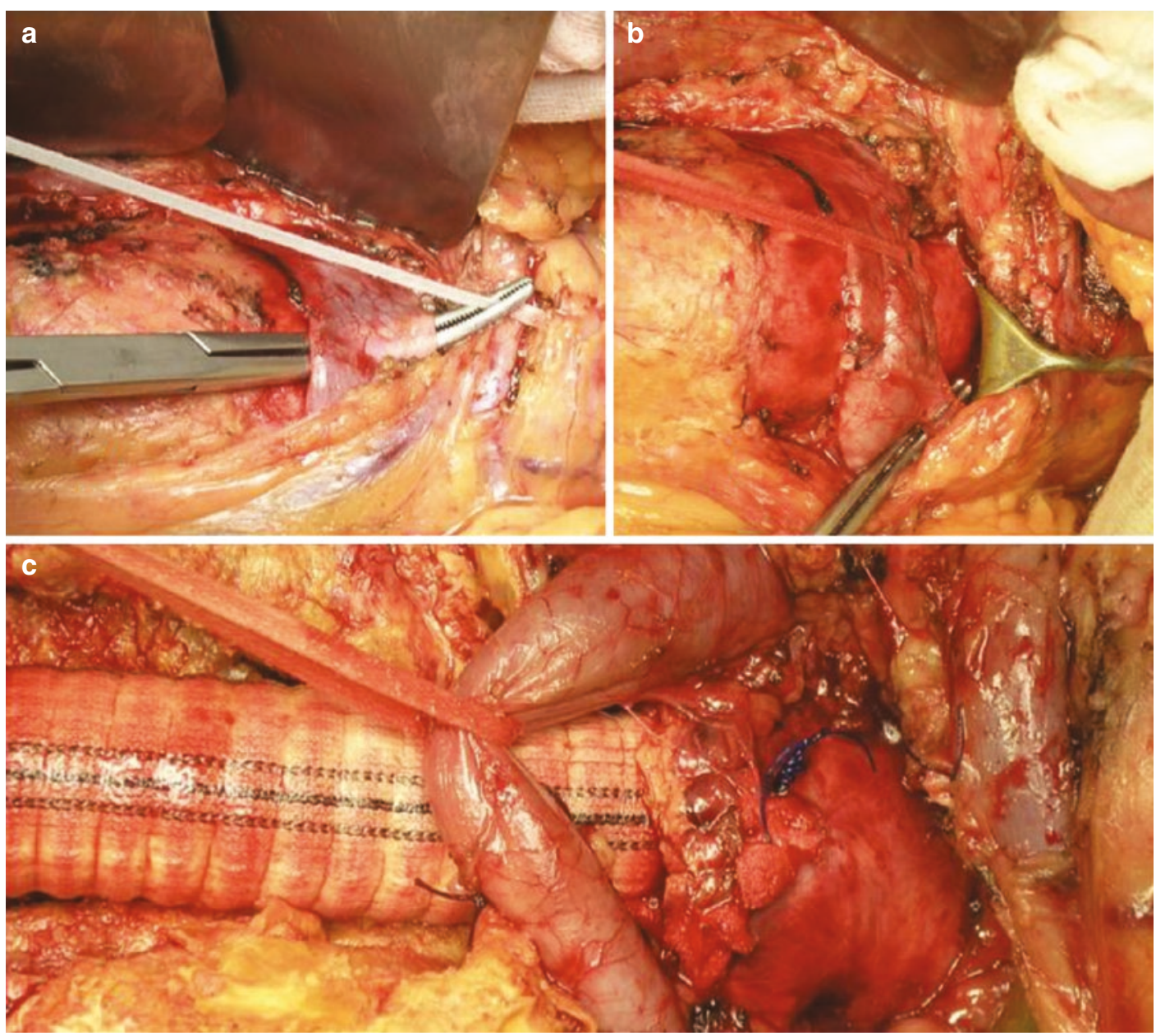

Fig. 3.3 Intraoperative photographs showing the correct steps to mobilize the left renal vein without damaging it
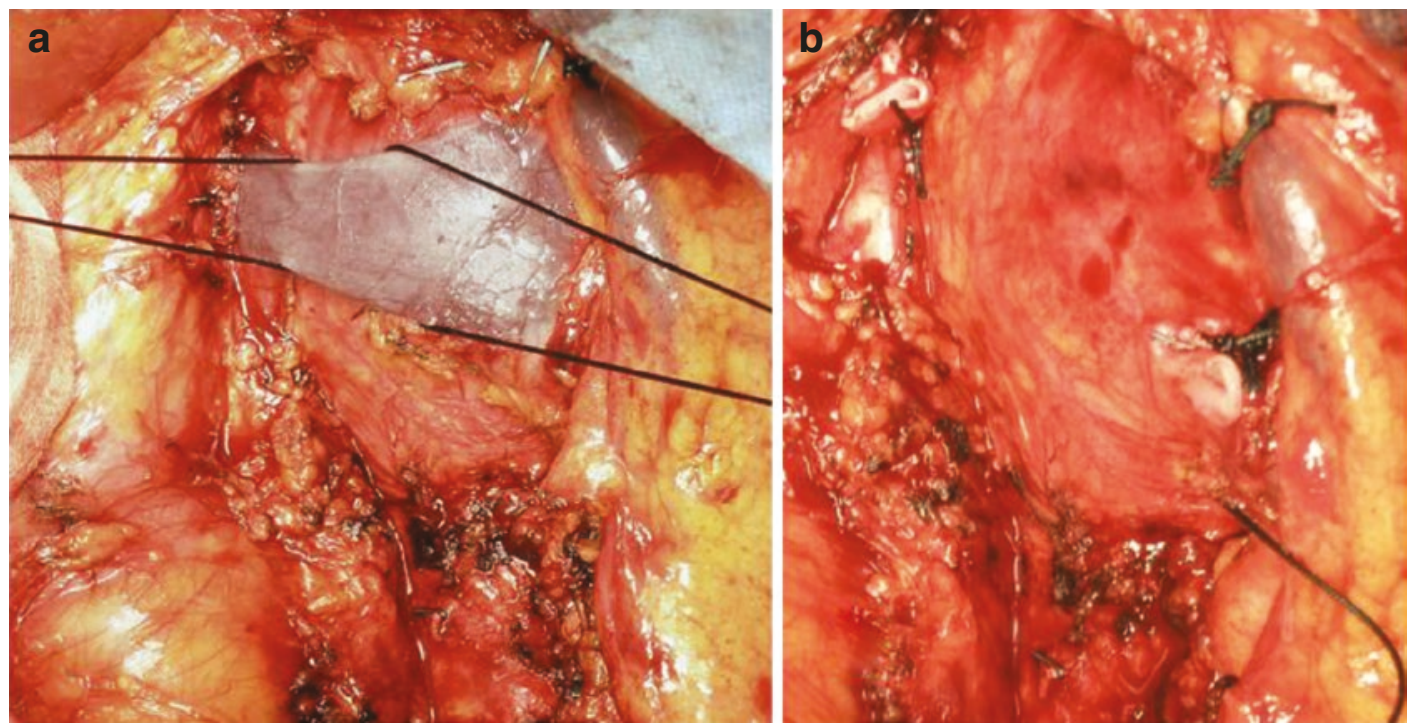

Fig. 3.4 Intraoperative photographs showing the correct steps to section the left renal vein 

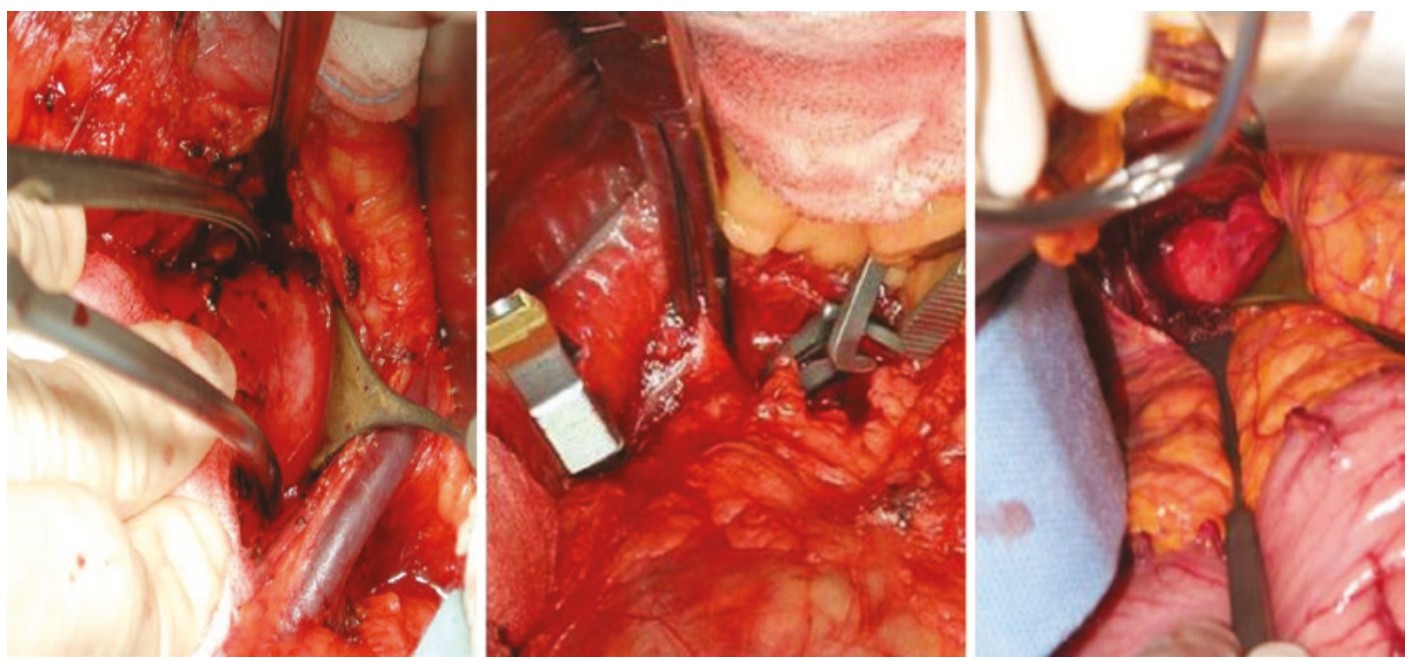

Fig. 3.5 Different clamping sites. From left to right: infrarenal, suprarenal, supra-celiac
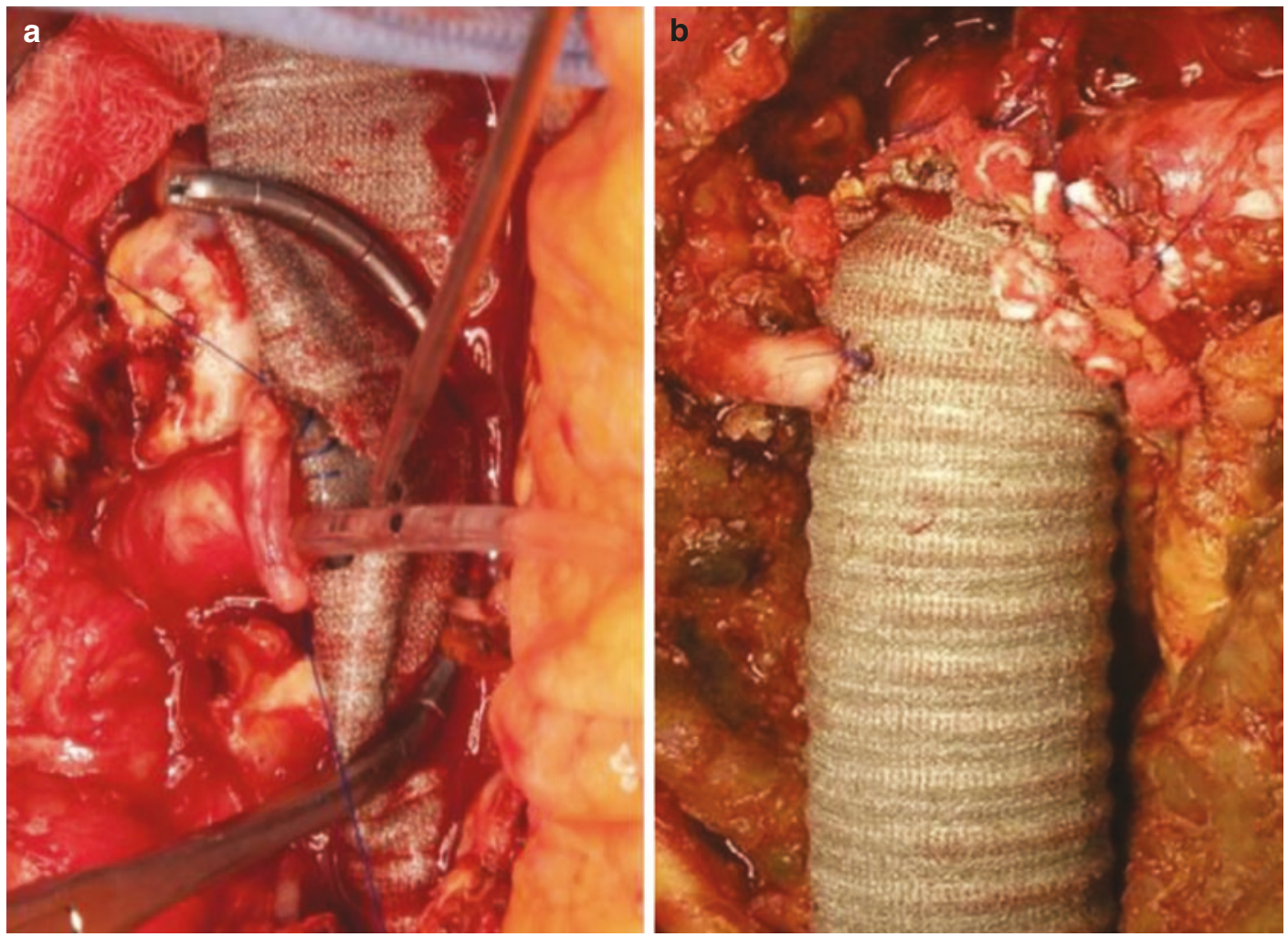

Fig. 3.6 Intraoperative pictures showing (a) the right renal artery being perfused while the anastomosis to the aortic graft is completed and (b) the final result of a direct renal artery reattachment to the aortic graft

In the case of renal artery involvement with no option of infrarenal aortic reconstruction, we put various strategies in place:
- Aorto-aortic repair with proximal end-to-end anastomosis and direct renal reattachment to the aortic graft (Fig. 3.6) 

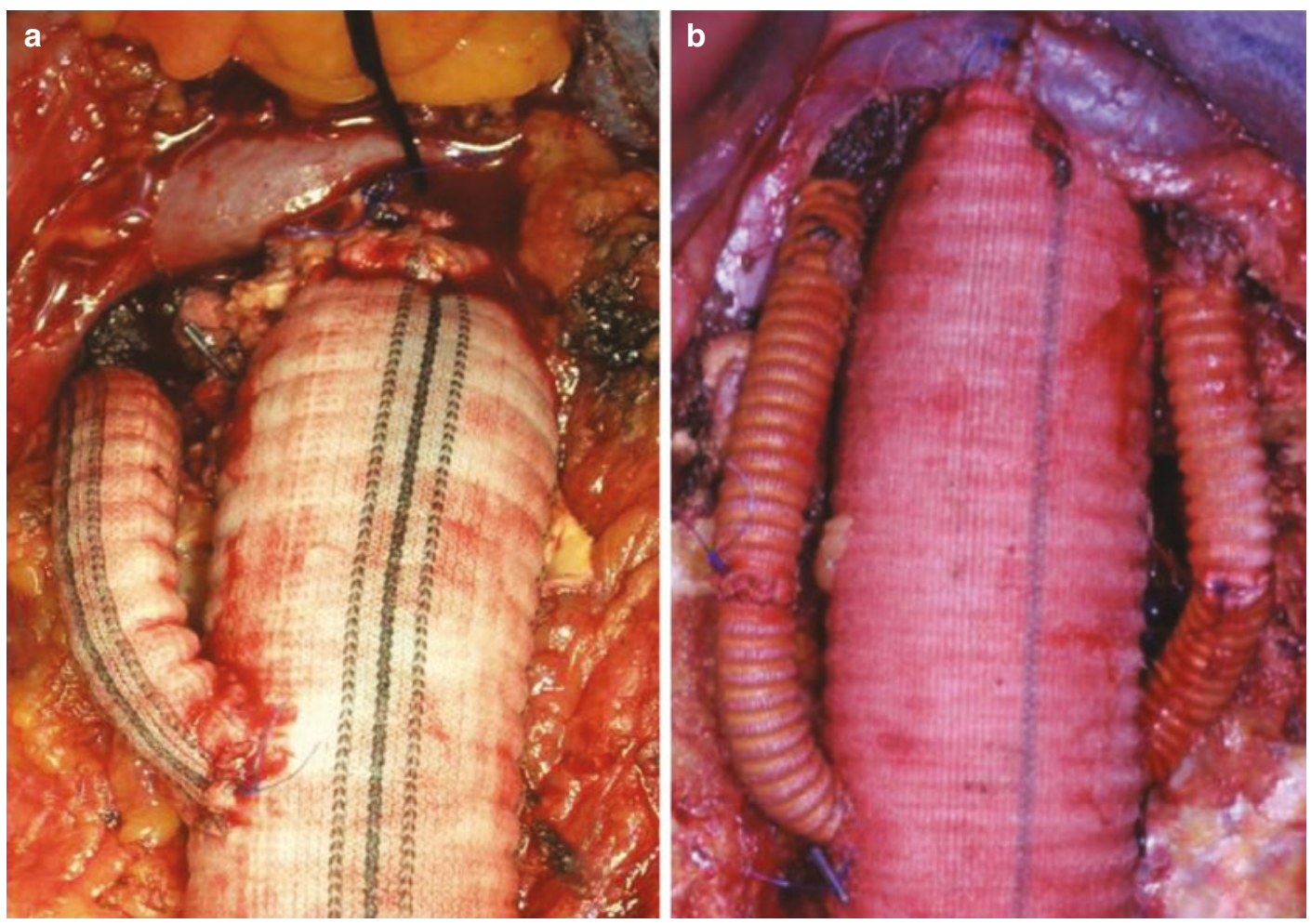

Fig. 3.7 Intraoperative pictures showing aorto-aortic repair with proximal end-to-end anastomosis and (a) uni- or (b) bilateral aorto-renal bypass grafting

- Aorto-aortic repair with proximal end-to-end anastomosis and uni- or bilateral aorto-renal bypass grafting (Fig. 3.7)

- Aorto-aortic repair with proximal end-to-end anastomosis and uni- or bilateral aorto-renal bypass grafting using a Gore Hybrid graft, inserting the stent in the side into the renal artery, deploying it, and then reinforcing it with four single sutures (Figs. 3.8 and 3.9)

- A proximal beveled anastomosis that includes renal arteries

- Carrel's patch including visceral vessels (celiac trunk, superior mesenteric, and renal arteries) that may be performed by means of a thoracophreno-laparotomy or through bilateral subcostal laparotomy with medial visceral rotation

\subsection{Complications}

The perioperative complications are not rare events in the surgical repair of the P-AAAs. It is clear that the perioperative management is crucial

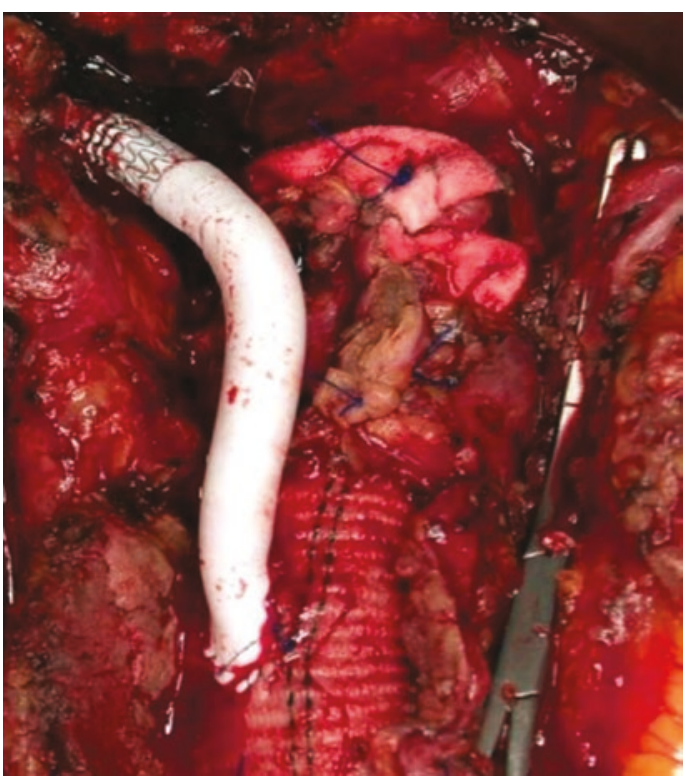

Fig. 3.8 Aorto-aortic repair with proximal end-to-end anastomosis and aorto-renal bypass grafting using a Gore Hybrid graft, inserting the stent in the side into the renal artery, deploying it, and then reinforcing it with four single sutures 

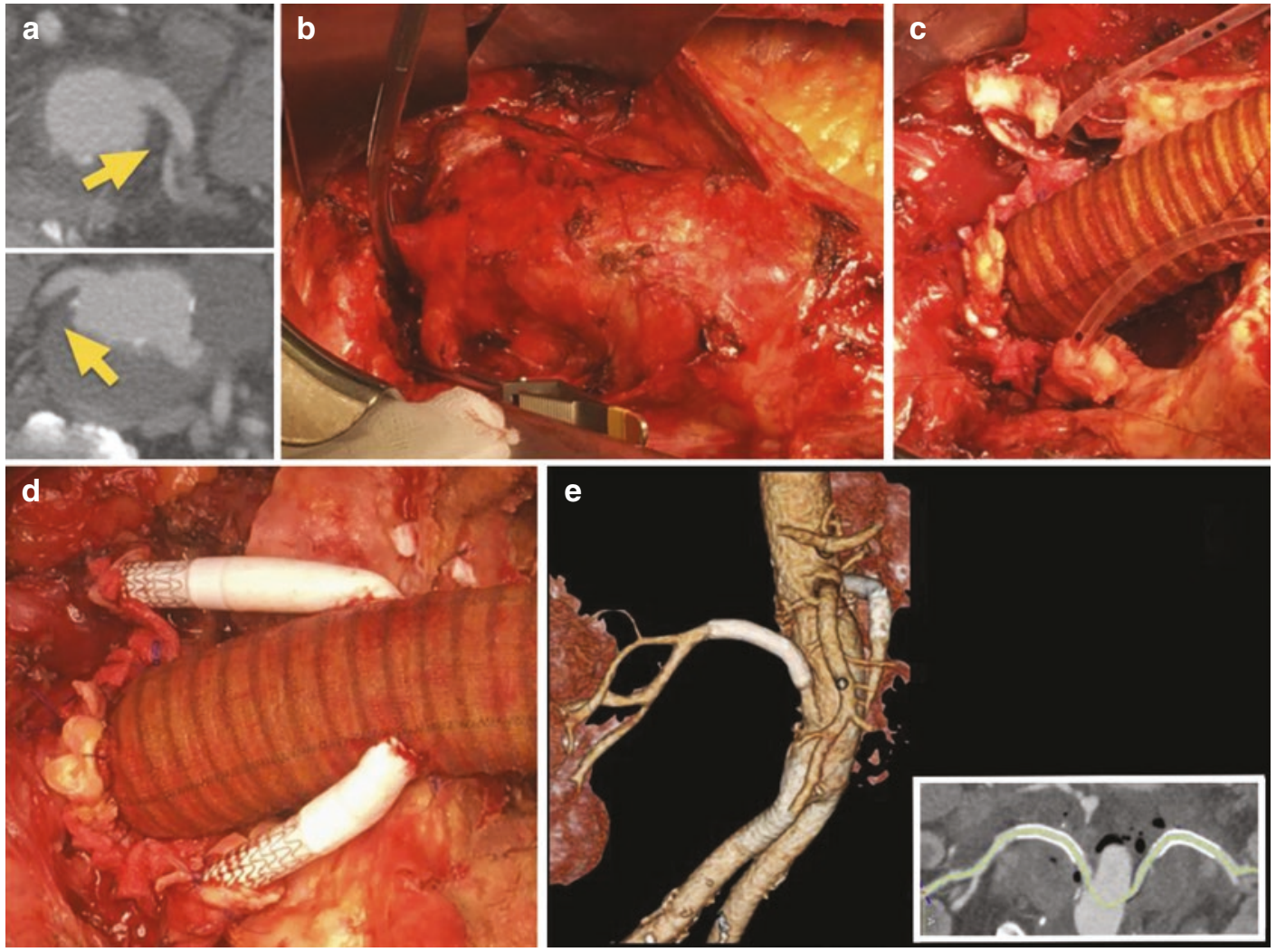

Fig. 3.9 (a) Preoperative CT scan showing thrombus close to the ostium of the renal arteries and a dissection of the right renal artery, (b) intraoperative picture showing the juxtarenal aortic aneurysm, (c) intraoperative picture showing the end-to-end proximal anastomosis and the two

in reducing postoperative complications. A team of professionals is needed with experience in this type of work, not only from a strictly surgical point of view but also anesthetic and nursing [28, 29].

\subsubsection{Bleeding}

Because of the type of aneurysmectomy P-AAAs, patients are subject to increased risk of bleeding in the perioperative period. Meticulous attention to hemostasis is essential to minimize the risk of postoperative bleeding, and the surgeon must pay close attention to the fact that there is no active bleeding before closing the retroperitoneum or abdomen. All anastomoses must be checked and all surfaces thoroughly examined. The therapy with blood components should be used to correct renal arteries being selectively perfused, (d) intraoperative picture showing renal artery reconstruction with a Gore Hybrid bypass graft, (e) postoperative CT scan showing the renal arteries reimplanted on the graft using the Gore Hybrid bypass graft

the coagulopathy that inevitably occurs. Similarly, topical hemostatic agents can be of great value in treating bleeding of the anastomosis. Despite all, postoperative bleeding can still occur.

The causes of bleeding can be:

- Bleeding from the anastomosis

- Dripping from the wall

- Bleeding from retrograde lumbar branches inadequately controlled

- Insult spleen, often secondary to the use of retractors

\subsubsection{Cardiac ischemia}

As described above, aortic clamping causes significant hemodynamic disturbances and creates an 
increase in cardiac workload. Preoperative screening, risk stratification, and appropriate patient selection may help to reduce this. The use of betablockers, in the perioperative period, in patients undergoing non-high-risk cardiac surgery, has been shown to reduce cardiovascular events and mortality at 30 days [30-32]. The administration of beta-blockers is associated with a significant reduction in peri- and postoperative mortality [33].

\subsubsection{Kidney Failure}

Patients undergoing surgical repair of P-AAA have a significant risk of developing kidney complications (Figs. 3.10 and 3.11). The percentage of postoperative renal failure in Medicare rises to $10 \%$ study, although with only $0.5 \%$ needing renal replacement therapy [34]. A meta-analysis of patients undergoing elective surgery for aortic pararenal aneurysmectomy showed the presence of postoperative renal failure in $15-20 \%$ of patients, of which only $3.5 \%$ require dialysis [35]. The acute tubular necrosis secondary to renal hypoperfusion is the leading cause of acute renal failure in these patients and occurs because of the important hemodynamic changes that the aortic clamping causes to the renal blood flow, as well as hypovolemia and perioperative hypotension. Particular attention should be paid to blood volume and to the use of crystalloid and colloid during the perioperative period as they are essential in the prevention of recurrent episodes of renal ischemia and for tackling the negative effects of aortic clamping. Despite the evidence that few patients need continuous renal replacement
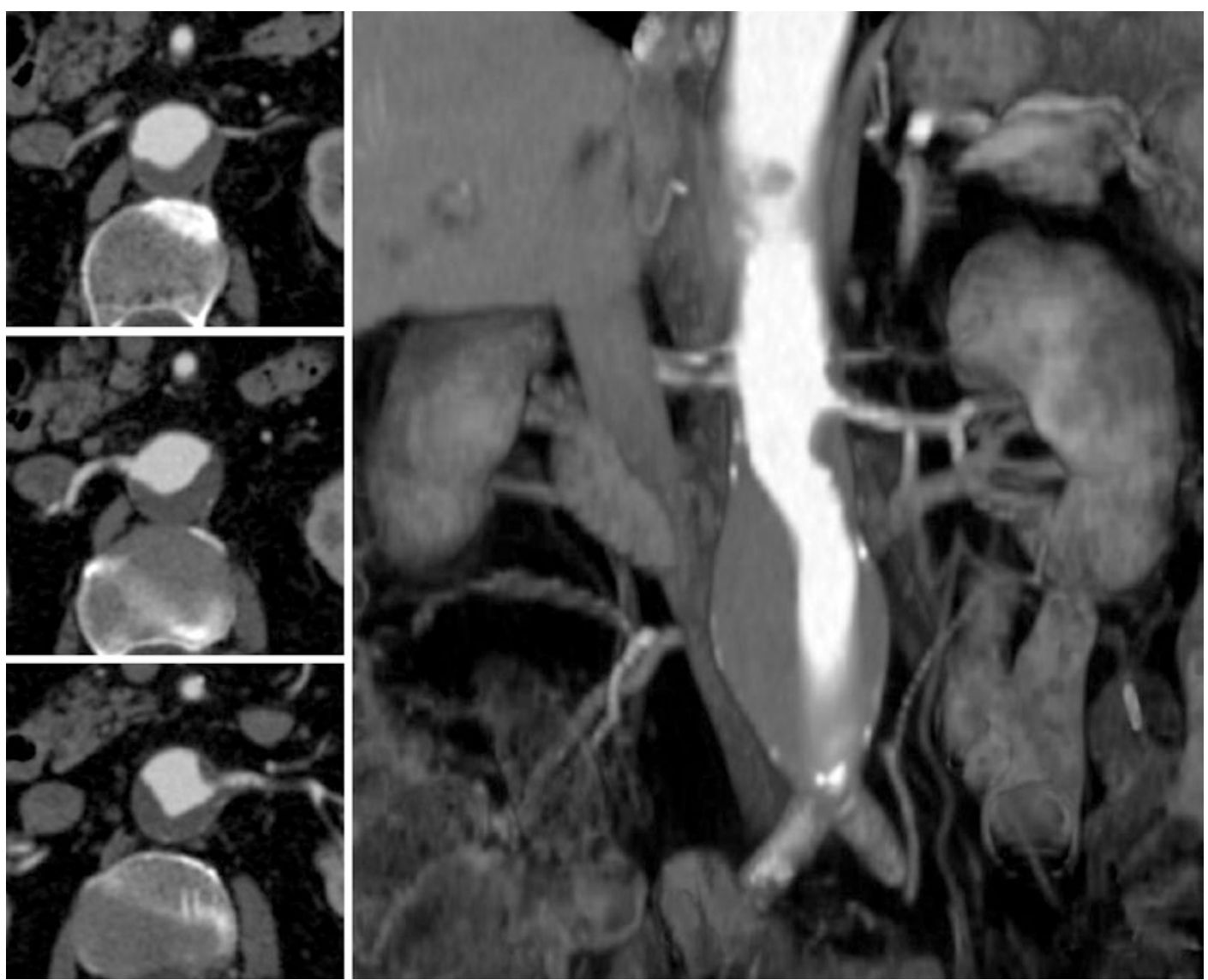

Fig. 3.10 Preoperative CT scan showing parietal thrombus close to the origin of the renal arteries 

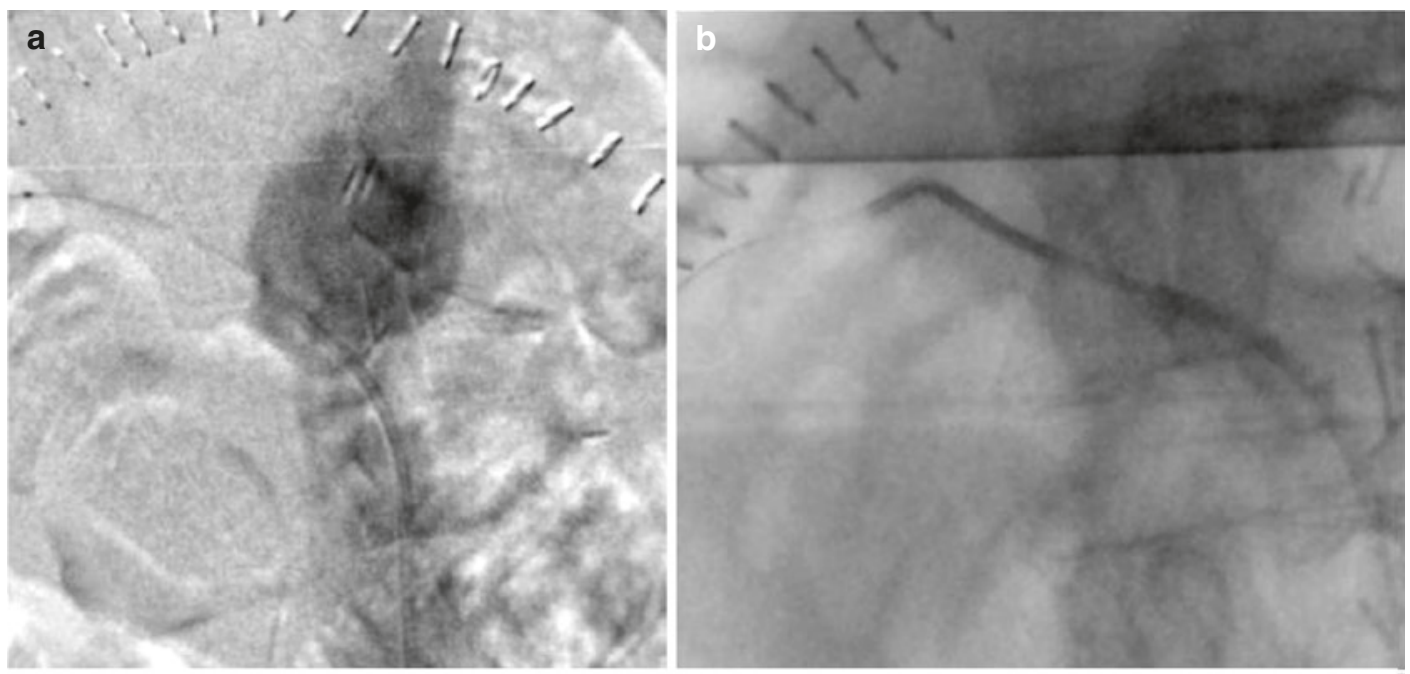

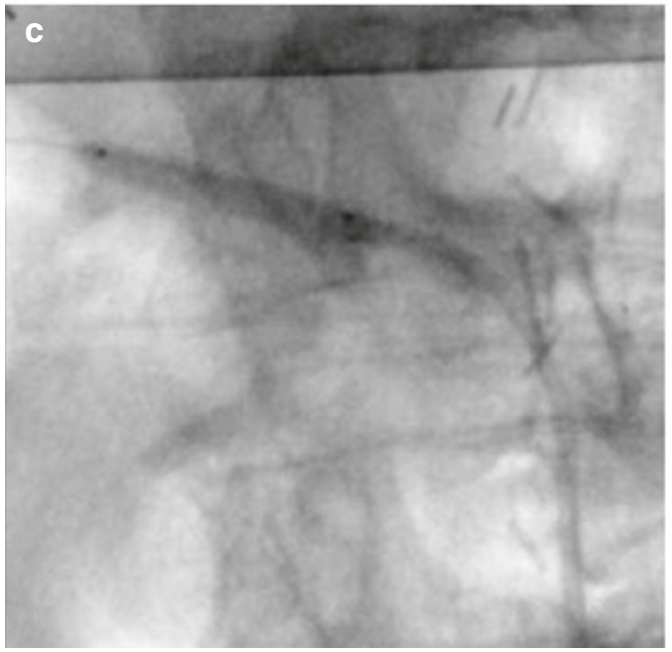

Fig. 3.11 Intraoperative angiography showing (a) complete occlusion of both right and left renal arteries, (b) catheterization of the right renal artery with a BERN

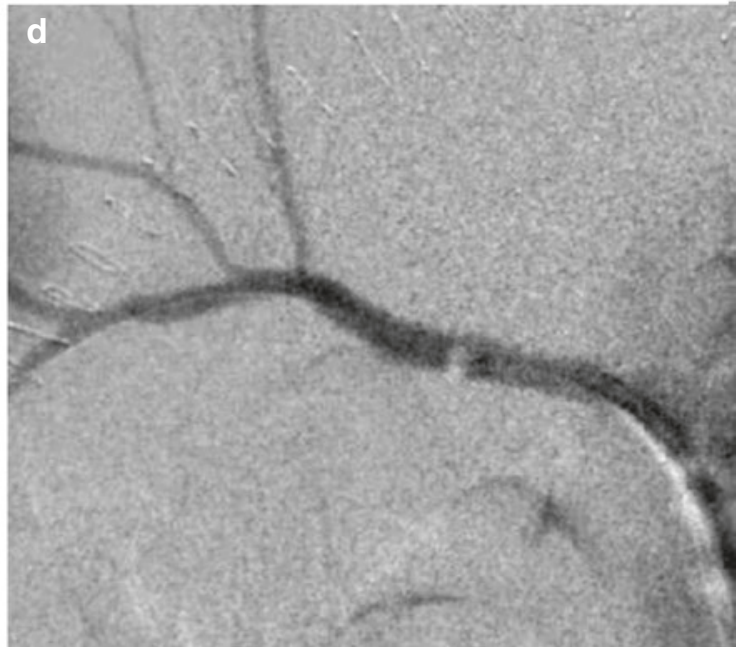

catheter, (c) ballooning of the vessels, and (d) complete angiography which shows restoration of the blood flow to the right kidney therapy, it has been shown that even subtle changes in the glomerular filtration rate can have profound long-term effects. The postoperative renal failure, in fact, has been shown to adversely affect the short- and long-term survival after this kind of surgery [36, 37].

\subsubsection{Classification and Staging of Acute Kidney Injury (AKI)}

The KDIGO (Kidney Disease Improving Global Outcomes) guidelines were released in 2012; they associated with the RIFLE (Risk, Injury, Failure, Loss, End-Stage Kidney Disease) and AKIN (Acute Kidney Injury Network) criteria. The KDIGO guidelines introduced a new definition of AKI and a new kidney injury staging system: AKI was defined as an increase in serum creatinine of $0.3 \mathrm{mg} / \mathrm{dL}$ or more within $48 \mathrm{~h}$ of surgery, or an increase of at least 1.5 times compared to the preoperative value in the last 7 days, or a lower hourly diuresis of $0.5 \mathrm{~mL} / \mathrm{kg}$ for more than $6 \mathrm{~h} \mathrm{[38].}$ 


\subsubsection{Intestinal Ischemia}

Intestinal ischemia is a feared complication in the surgical repair of abdominal aortic aneurysm. Binding of the AMI, failure of the hypogastric artery revascularization, iliofemoral occlusive disease, stenosis of the SMA, athero-embolism, injuries from retractor, and previous resection of the colon may contribute to intestinal ischemia, which has a clinical incidence from $0.2 \%$ to $6 \%$ following surgical repair of AAA [17, 39, 40]. Upon diagnosis, intestinal ischemia requires early and aggressive treatment because it has a generally unfavorable outcome. In some large series, the intestinal ischemia is associated with mortality rates ranging from $25 \%$ to $50 \%$ [40, 41]. Consequently, all patients should be treated aggressively with intravenously broad-spectrum antibiotics, targeting the bacteria of the intestinal flora. Patients with full-thickness ischemia require emergency treatment with exploratory laparotomy and bowel resection [42].

\subsubsection{Long-Term Complications}

There is clear evidence from many sources that the proximal aorta continues to reshape over time, regardless of the type of repair [18]. Although the anastomosis with continuous suture allows a robust surgical fixation of the aortic graft, a possible long-term complication is represented by anastomotic pseudoaneurysms. Large cohorts with a significant follow-up pose the incidence of anastomotic pseudoaneurysms to $<1 \%$ in 6 years, from $1 \%$ to $10 \%$ at 10 years, and $20 \%$ at 15 years, depending on the study [19, 20, 43, 44]. One of these studies, the Mayo Clinic, is significant because it followed 307 patients undergoing AAA repair surgery for an average of 5.8 cases by years [20]. The anastomotic pseudoaneurysm is the most common late complication, with a median of 6 years of onset period; other complications are occlusion $(2 \%)$, aorto-enteric fistula (1.6\%), and the prosthesis infection $(1.3 \%)$. Taken together, these data show that the result of a correct surgical repair of abdominal aortic aneurysm is excellent, but not perfect. Although the surgery definitively repairs the aneurysmal segment, the underlying aortic disease remains, and this leads to dilation of the proximal and distal portion. This can cause the formation of metachronous aneurysms and pseudoaneurysms, as well as increase the likelihood of rupture of the anastomosis. For these reasons, all patients who underwent surgical repair of abdominal aortic aneurysm require at least a permanent ultrasound monitoring.

\section{References}

1. Chaikof EL, Blankensteijn JD, Harris PL, et al. Reporting standards for endovascular aortic aneurysm repair. J Vasc Surg. 2002;35(5):1048-60. https://doi. org/10.1067/mva.2002.123763.

2. Back MR, Bandyk M, Bradner M, et al. Critical analysis of outcome determinants affecting repair of intact aneurysms involving the visceral aorta. Ann Vasc Surg. 2005;19(5):648-56. https://doi.org/10.1007/ s10016-005-6843-3.

3. Gillum RF. Epidemiology of aortic aneurysm in the United States. J Clin Epidemiol. 1995;48(11):128998. https://doi.org/10.1016/0895-4356(95)00045-3.

4. Katz DJ, Stanley JC, Zelenock GB. Operative mortality rates for intact and ruptured abdominal aortic aneurysms in Michigan: an eleven-year statewide experience. J Vasc Surg. 1994;19(5):80417. https://doi.org/10.1016/S0741-5214(94)70005-2.

5. LaMorte WW, Scott TE, Menzoian JO. Racial differences in the incidence of femoral bypass and abdominal aortic aneurysmectomy in Massachusetts: relationship to cardiovascular risk factors. J Vasc Surg. 1995;21(3):422-31. https://doi.org/10.1016/ S0741-5214(95)70284-9.

6. Wilmink ABM, Hubbard CS, Day NE, Quick CRG. The incidence of small abdominal aortic aneurysms and the change in normal infrarenal aortic diameter: implications for screening. Eur J Vasc Endovasc Surg. 2001;21(2):165-70. https://doi. org/10.1053/ejvs.2000.1285.

7. Melton LJ, Bickerstaff LK, Hollier LH, et al. Changing incidence of abdominal aortic aneurysms: a population-based study. Am J Epidemiol. 1984;120(3):379-86. https://doi.org/10.1093/oxfordjournals.aje.a113902.

8. Wilmink ABM, Quick CRG. Epidemiology and potential for prevention of abdominal aortic aneurysm. Br J Surg. 1998;85(2):155-62. https://doi. org/10.1046/j.1365-2168.1998.00714.x.

9. Lederle FA, Johnson GR, Wilson SE, et al. Prevalence and associations of abdominal aortic aneurysm detected through screening. Aneurysm detection and management (ADAM) veterans affairs cooperative 
study group. Ann Intern Med. 1997;126(6):441-9. https://doi.org/10.1056/NEJM200212053472325.

10. Moll F, Powell J, Fraedrich G, et al. Management of abdominal aortic aneurysms clinical practice guidelines of the European society for vascular surgery. Eur J Vasc Endovasc Surg. 2011;41(S1):S1-S58. https:// doi.org/10.1016/j.ejvs.2010.09.011.

11. Juvonen T, Ergin MA, Galla JD, et al. Risk factors for rupture of chronic type B dissections. J Thorac Cardiovasc Surg. 1999;117(4):776-86. https://doi. org/10.1016/S0022-5223(99)70299-0.

12. Juvonen T, Ergin MA, Galla JD, et al. Prospective study of the natural history of thoracic aortic aneurysms. Ann Thorac Surg. 1997;63(6):1533-45. https://doi.org/10.1016/S0003-4975(97)00414-1.

13. Lo RC, Lu B, Fokkema MTM, et al. Relative importance of aneurysm diameter and body size for predicting abdominal aortic aneurysm rupture in men and women. J Vasc Surg. 2014;59(5):1209-16. https://doi. org/10.1016/j.jvs.2013.10.104.

14. Beales L, Wolstenhulme S, Evans JA, West R, Scott DJA. Reproducibility of ultrasound measurement of the abdominal aorta. Br J Surg. 2011;98(11):1517-25. https://doi.org/10.1002/bjs.7628.

15. Shuman W, Hastrup W, Kohler T, et al. Suspected leaking abdominal aortic aneurysm: use of sonography in the emergency room. Radiol Clin N Am. 1988;168(1):117-9. https://doi.org/10.1148/ radiology.168.1.3289085.

16. Pavone P, Di Cesare E, Di Renzi P, et al. Abdominal aortic aneurysm evaluation: comparison of US, CT, MRI, and angiography. Magn Reson Imaging. 1990;8(3):199-204. https://doi. org/10.1016/0730-725X(90)90089-K.

17. Becquemin J-P, Majewski M, Fermani N, et al. Colon ischemia following abdominal aortic aneurysm repair in the era of endovascular abdominal aortic repair. J Vasc Surg. 2008;47(2):258-63; discussion 263. https://doi.org/10.1016/j.jvs.2007.10.001.

18. Cao P, De Rango P, Parlani G, Verzini F. Fate of proximal aorta following open Infrarenal aneurysm repair. Semin Vasc Surg. 2009;22(2):93-8. https://doi. org/10.1053/j.semvascsurg.2009.04.005.

19. Edwards JM, Teefey SA, Zierler RE, Kohler TR. Intraabdominal paraanastomotic aneurysms after aortic bypass grafting. J Vasc Surg. 1992;15(2):34453. https://doi.org/10.1016/0741-5214(92)90256-8.

20. Hallett J, Marshall DM, Petterson TM, et al. Graftrelated complications after abdominal aortic aneurysm repair: reassurance from a 36-year population-based experience. J Vasc Surg. 1997;25(2):277-86. https:// doi.org/10.1016/S0741-5214(97)70349-5.

21. Sun ZH. Abdominal aortic aneurysm: treatment options, image visualizations and follow-up procedures. J Geriatr Cardiol. 2012;9(1):49-60. https://doi. org/10.3724/SP.J.1263.2012.00049.

22. Squillaci E, Fanucci E, Masala S, Nisini A, Tomassini M, Simonetti G. A comparison between two different concentration of contrast media with multidetec- tor CT for the study of abdominal vascular system. Radiol Med. 2002;104(4):341-50.

23. Van Kuijk JP, Flu WJ, Dunckelgrun M, Bax JJ, Poldermans D. Coronary artery disease in patients with abdominal aortic aneurysm: a review article. J Cardiovasc Surg. 2009;50(1):93-107.

24. Poldermans D, Bax JJ, Boersma E, et al. Guidelines for pre-operative cardiac risk assessment and perioperative cardiac management in non-cardiac surgery. Eur Heart J. 2009;30(22):2769-812. https://doi. org/10.1093/eurheartj/ehp337.

25. Thomas D, Anderson D, Hulten E, et al. Open versus endovascular repair of abdominal aortic aneurysm: incidence of cardiovascular events in 632 patients in a department of defense cohort over 6-year follow-up. Vascular. 2014;0(0):1-6. https://doi. org/10.1177/1708538114546207.

26. Inker LA, Astor BC, Fox CH, et al. KDOQI US commentary on the 2012 KDIGO clinical practice guideline for the evaluation and management of CKD. Am J Kidney Dis. 2014;63(5):713-35. https:// doi.org/10.1053/j.ajkd.2014.01.416.

27. Cirillo M, Lombardi C, Mele AA, Marcarelli F, Bilancio G. A population-based approach for the definition of chronic kidney disease: the CKD prognosis consortium. J Nephrol. 2012;25(1):7-12. https://doi. org/10.5301/jn.5000045.

28. Pronovost P, Jenckes M, Dorman T, Garrett E. Organizational characteristics of intensive care units related to outcomes of abdominal aortic surgery. JAMA. 1999;281(14):1310-7. https://doi. org/10.1001/jama.281.14.1310.

29. Pronovost PJ, Dang D, Dorman T, et al. Intensive care unit nurse staffing and the risk for complications after abdominal aortic surgery. Eff Clin Pract. 2001;4(5):199-206. http://www.ncbi.nlm.nih.gov/ pubmed/11685977

30. Lindenauer PK, Pekow P, Wang K, Mamidi DK, Gutierrez B, Benjamin EM. Perioperative betablocker therapy and mortality after major noncardiac surgery. N Engl J Med. 2005;353(4):349-61. https:// doi.org/10.1056/NEJMoa041895.

31. Poldermans D, Boersma E, Bax JJ, et al. The effect of bisoprolol on perioperative mortality and myocardial infarction in high-risk patients undergoing vascular surgery. Dutch echocardiographic cardiac risk evaluation applying stress echocardiography study group. N Engl J Med. 1999;341(24):1789-94. https://doi. org/10.1056/NEJM199912093412402.

32. Mangano DT, Layug EL, Wallace A, Tateo I. Effect of atenolol on mortality and cardiovascular morbidity after noncardiac surgery. Multicenter study of perioperative ischemia research group. $\mathrm{N}$ Engl J Med. 1996;335(23):1713-20. https://doi.org/10.1056/ NEJM199612053352301.

33. Kurzencwyg D, Filion KB, Pilote L, et al. Cardiac medical therapy among patients undergoing abdominal aortic aneurysm repair. Ann Vasc Surg. 2006;20(5):569-76. https://doi.org/10.1007/ s10016-006-9078-z. 
34. Schermerhorn ML, O’Malley AJ, Jhaveri A, Cotterill P, Pomposelli F, Landon BE. Endovascular vs. open repair of abdominal aortic aneurysms in the Medicare population. N Engl J Med. 2008;358(5):464-74. https://doi.org/10.1056/NEJMoa0707348.

35. Tallarita T, Sobreira ML, Oderich GS. Results of open pararenal abdominal aortic aneurysm repair: tabular review of the literature. Ann Vasc Surg. 2011;25(1):143-9. https://doi.org/10.1016/j. avsg.2010.10.005.

36. Nathan DP, Brinster CJ, Woo EY, Carpenter JP, Fairman RM, Jackson BM. Predictors of early and late mortality following open extent IV thoracoabdominal aortic aneurysm repair in a large contemporary singlecenter experience. J Vasc Surg. 2011;53(2):299-306. https://doi.org/10.1016/j.jvs.2010.08.085.

37. Grant SW, Grayson AD, Grant MJ, Purkayastha D, McCollum CN. What are the risk factors for renal failure following open elective abdominal aortic aneurysm repair? Eur J Vasc Endovasc Surg. 2012;43(2):182-7. https://doi.org/10.1016/j.ejvs.2011.11.018.

38. Mehta RL, Kellum JA, Shah SV, et al. Acute kidney injury network: report of an initiative to improve outcomes in acute kidney injury. Crit Care. 2007;11(2):R31. https://doi.org/10.1186/cc5713.

39. Bjorck M, Troeng T, Bergqvist D. Risk factors for intestinal ischaemia after aortoiliac surgery: a com- bined cohort and case-control study of 2824 operations. Eur J Vasc Endovasc Surg. 1997;13(6):531-9. https://doi.org/10.1016/S1078-5884(97)80061-5.

40. Brewster DC, Franklin DP, Cambria RP, et al. Intestinal ischemia complicating abdominal aortic surgery. Surgery. 1991;109(4):447-54. http://www. ncbi.nlm.nih.gov/pubmed/1844392

41. Longo WE, Lee TC, Barnett MG, et al. Ischemic colitis complicating abdominal aortic aneurysm surgery in the U.S. veteran. J Surg Res. 1996;60(2):351-4. https://doi.org/10.1006/jsre.1996.0056.

42. Menegaux F, Trésallet C, Kieffer E, Bodin L, Thabut D, Rouby J-J. Aggressive management of nonocclusive ischemic colitis following aortic reconstruction. Arch Surg. 2006;141(7):678-82. https://doi. org/10.1016/S0749-4041(08)70719-X.

43. Hertzer NR, Mascha EJ, Karafa MT, O'Hara PJ, Krajewski LP, Beven EG. Open infrarenal abdominal aortic aneurysm repair: the Cleveland Clinic experience from 1989 to 1998. J Vasc Surg. 2002;35(6):114554. https://doi.org/10.1067/mva.2002.123686.

44. Kalman PG, Rappaport DC, Merchant N, Clarke $\mathrm{K}$, Johnston KW. The value of late computed tomographic scanning in identification of vascular abnormalities after abdominal aortic aneurysm repair. J Vasc Surg. 1999;29(3):442-50. https://doi.org/10.1016/ S0741-5214(99)70272-7. 